\title{
Logarithmic Boundary Layers in Strong Taylor-Couette Turbulence
}

\author{
Sander G. Huisman, ${ }^{1}$ Sven Scharnowski, ${ }^{2}$ Christian Cierpka, ${ }^{2}$ Christian J. Kähler, ${ }^{2}$ Detlef Lohse, ${ }^{1}$ and Chao Sun ${ }^{1}$ \\ ${ }^{1}$ Department of Applied Physics and J. M. Burgers Centre for Fluid Dynamics, University of Twente, \\ P.O. Box 217, 7500 AE Enschede, The Netherlands \\ ${ }^{2}$ Institut für Strömungsmechanik und Aerodynamik, Universität der Bundeswehr München, \\ Werner-Heisenberg-Weg 39, 85577 Neubiberg, Germany \\ (Received 22 February 2013; published 26 June 2013)
}

\begin{abstract}
We provide direct measurements of the boundary layer properties in highly turbulent Taylor-Couette flow up to $\mathrm{Re}=2 \times 10^{6}\left(\mathrm{Ta}=6.2 \times 10^{12}\right)$ using high-resolution particle image velocimetry and particle tracking velocimetry. We find that the mean azimuthal velocity profile at the inner and outer cylinder can be fitted by the von Kármán $\log \operatorname{law} u^{+}=1 / \kappa \ln y^{+}+B$. The von Kármán constant $\kappa$ is found to depend on the driving strength Ta and for large Ta asymptotically approaches $\kappa \approx 0.40$. The variance profiles of the local azimuthal velocity have a universal peak around $y^{+} \approx 12$ and collapse when rescaled with the driving velocity (and not with the friction velocity), displaying a log dependence of $y^{+}$as also found for channel and pipe flows.
\end{abstract}

DOI: 10.1103/PhysRevLett.110.264501

PACS numbers: 47.27.nb, 47.32.Ef

Taylor-Couette (TC) flow is one of the paradigmatical flows in physics of fluids next to Rayleigh-Bénard (RB) convection, channel, flat plate, and pipe flow. It consists of two rotating coaxial cylinders shearing a fluid in between the cylinders, see Fig. 1. For only inner cylinder rotation, the Reynolds number $\operatorname{Re}=\omega_{i} r_{i}\left(r_{o}-r_{i}\right) / \nu$ quantifies the driving of this system. It is closely connected to the Taylor number $\mathrm{Ta}=1 / 4(1+\eta / 2 \sqrt{\eta})^{4}\left(r_{o}-r_{i}\right)^{2}\left(r_{i}+r_{o}\right)^{2} \omega_{i}^{2} / \nu^{2}$, the ratio of centrifugal forces to viscous forces. Here, $\omega=$ $u_{\theta} / r$ is the angular velocity component, $r$ the radius, $\nu$ the kinematic viscosity, $\eta=r_{i} / r_{o}$ the radius ratio, and $i$ and $o$ subscripts denote quantities related to the inner and outer cylinder, respectively. For increasing Re, the system is first dominated by coherent structures [1] whose length scale is of similar size as the gap width. For further increasing Re, turbulence develops in the bulk at length scales between the integral and the Kolmogorov scale while the boundary layers (BL) are still of laminar type. This regime, in which the flow has a turbulent bulk and the boundary layers are of Prandtl-Blasius type, is called the classical regime [2]. By further increasing $\mathrm{Re}$, the system enters the so-called ultimate turbulent state in which also the boundary layers have turned turbulent [3-6]. Note that vortical structures appear at the first transition above a critical Ta [7] and continue to persist within the ultimate regime [8,9]. Based on global transport measurements, the ultimate regime of turbulence sets in at $\operatorname{Re}(\mathrm{Ta}) \sim 10^{4}\left(10^{8}\right)$ $[8,10,11]$.

The coexistence of a laminar-type boundary layer and turbulent bulk in classical turbulent RB convection has been well established from numerous experimental and numerical investigations [12-15]. This is also the case for the classical regime in TC flow [8,16-19] and the transition regime to ultimate TC flow [20-22]. Recently, a direct measurement [23] of the mean temperature profile close to the wall in the ultimate $\mathrm{RB}$ state revealed logarithmic behavior in the ultimate regime. For pipe, flat plate, and channel flows, numerous experiments have revealed the existence of a $\log$ layer for the velocity and its variance, see, e.g., Refs. [24-28].

Neither for TC nor for RB had there been any direct and systematical measurement of the velocity BL in the highly turbulent ultimate state due to the experimental difficulties. Assuming a logarithmic velocity profile in the boundary layers for highly turbulent TC flow, and matching the mean velocities at midgap, Lathrop et al. [10,29] obtained a dependence of the global torque and the Re, which agrees well with the torque measurements in the ultimate turbulence regime $[10,29,30]$. Only recently were direct

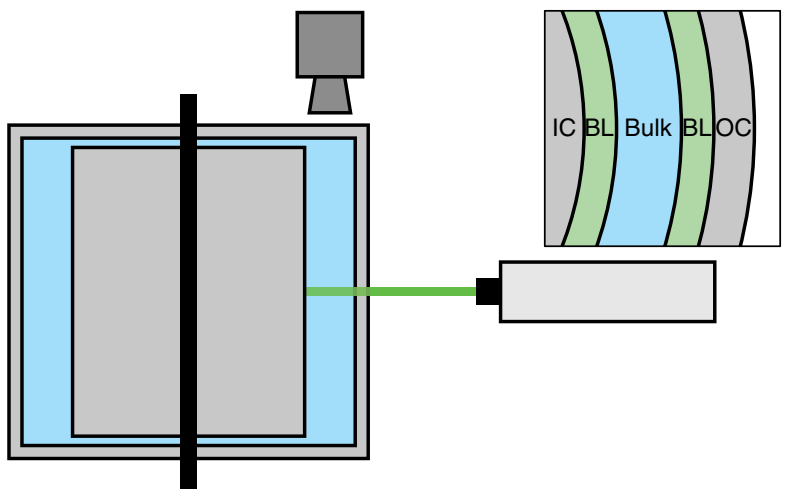

FIG. 1 (color online). Sketch of the vertical cross section of the $\mathrm{T}^{3} \mathrm{C}$ [39]. The flow is illuminated from the side in the horizontal plane using a laser, and the flow is imaged from the top using a high-resolution camera. Top-right inset: schematic top view of different regions inside the gap: IC (inner cylinder), OC (outer cylinder), and BL (boundary layer). Measurements were done at midheight. 
measurements on BLs conducted by van Hout and Katz [20] for Ta up to $2 \times 10^{9}$, where they focused on the effect of counterrotation and found that the von Kármán constant depends on the angular velocity ratio $\omega_{o} / \omega_{i}$.

In this Letter, we report the direct systematical experimental investigation of the boundary layer properties for very high $\mathrm{Ta}$ from $\mathrm{Ta}=9.9 \times 10^{8}$ to $\mathrm{Ta}=6.2 \times 10^{12}$ using high-resolution particle tracking velocimetry (PTV) and particle image velocimetry (PIV) [31-33] with an unprecedented spatial resolution down to $\approx 10 \mu \mathrm{m}$. We focus on the case of inner cylinder rotation, and examine the boundary layer properties as a function of $\operatorname{Re}(\mathrm{Ta})$ in the ultimate turbulent TC regime.

It was mathematically found [34] from the NavierStokes and continuity equations that $J_{\omega}=r^{3}\left(\left\langle u_{r} \omega\right\rangle_{z, \theta, t}-\right.$ $\left.\nu \partial_{r}\langle\omega\rangle_{z, \theta, t}\right)$ is strictly conserved in TC flow. Here $\langle X\rangle_{z, \theta, t}$ represents axial, azimuthal, and time averaging of $X$, and $u_{r}$ is the radial velocity. This transport quantity is independent of $r$; any flux going through an imaginary cylinder with radius $r$ also goes through any other imaginary cylinder, or mathematically $\partial_{r} J_{\omega}=0$. This flux can be measured locally $[30,35]$ but also globally $[10,36-38]$ by measuring the torque needed to sustain the constant velocity of the cylinders. The torque $\mathcal{T}$ is related to the dimensionless torque $G$ and to $J_{\omega}$ as follows:

$$
G=\frac{\mathcal{T}}{2 \pi \ell \rho \nu^{2}}=\frac{J_{\omega}}{\nu^{2}}
$$

where $\rho$ is the density of the fluid, and $\ell$ the height of the cylinders. We can further relate these quantities to the wall shear stress $\tau_{w}$, the friction velocity $u_{\tau}$, and the viscous length scale $\delta_{\nu}$ at the inner cylinder wall:

$$
\begin{gathered}
\tau_{w, i}=\mathcal{T} / 2 \pi r_{i}^{2} \ell, \\
u_{\tau, i}=\sqrt{\tau_{w, i} / \rho,} \\
\delta_{\nu, i}=\nu / u_{\tau, i} .
\end{gathered}
$$

Note that as $J_{\omega}$ is conserved radially, it is the same at both cylinders, and using Eq. (1), also the torque $\mathcal{T}$ at both cylinders is the same. Consequently, $\tau_{w}, u_{\tau}$, and $\delta_{\nu}$ are different at the inner and outer cylinder, and the following relations hold:

$$
\begin{gathered}
\tau_{w, i} / \tau_{w, o}=1 / \eta^{2}, \\
u_{\tau, i} / u_{\tau, o}=1 / \eta, \\
\delta_{\nu, i} / \delta_{\nu, o}=\eta .
\end{gathered}
$$

The apparatus used for the experiments, the Twente turbulent Taylor-Couette $\left(\mathrm{T}^{3} \mathrm{C}\right)$, has an inner cylinder with an outer radius of $r_{i}=0.200 \mathrm{~m}$ and a transparent outer cylinder with inner radius $r_{o}=0.279 \mathrm{~m}$ giving a radius ratio of $\eta=0.716$. The cylinders have a height of $\ell=0.927 \mathrm{~m}$ resulting in an aspect ratio of $\Gamma=$ $\ell /\left(r_{o}-r_{i}\right)=11.7$. More details can be found in Ref. [39]. For the PIV and PTV measurements, the working fluid (water) is seeded with fluorescent polymer particles [40]. Using a laser [41], we create a horizontal light sheet of roughly $500 \mu \mathrm{m}$ thickness for illumination. The flow is then imaged from the top (see Fig. 1) using a highresolution camera [42] with large dynamical range.

For each rotational velocity, $10^{4}$ image pairs were acquired at a recording frequency of $10 \mathrm{~Hz}$. The mean velocity distribution was computed using single-pixel ensemble correlation. This technique $[31,43]$ leads to a final resolution of $\approx 150 \mu \mathrm{m}$ and in over 500 independent data points in the $80 \mathrm{~mm}$ gap. The standard deviation was directly computed from the velocity probability density function, which was extracted from the shape of the correlation function, as discussed in Ref. [33]. This procedure ensures that all turbulent scales are included in the standard deviation. In contrast to standard PIV analysis, where only spatially low-pass filtered results are achieved, here also the contribution of the small-scale fluctuations is properly considered. In order to resolve the near-wall region at the inner cylinder, a microscope [44] was mounted in front of the camera. With this setup, a scaling factor of $\approx 10 \mu \mathrm{m} / \mathrm{px}$ was achieved. To maximize the spatial resolution, the near-wall region was evaluated with PTV methods, which are best suited for this purpose [32].

Figure 2 shows all the measured midheight profiles: five covering the full gap and two covering just the region near the inner cylinder. As expected, the profiles do not conform to the laminar nonvortical profile, and the bulk has a much

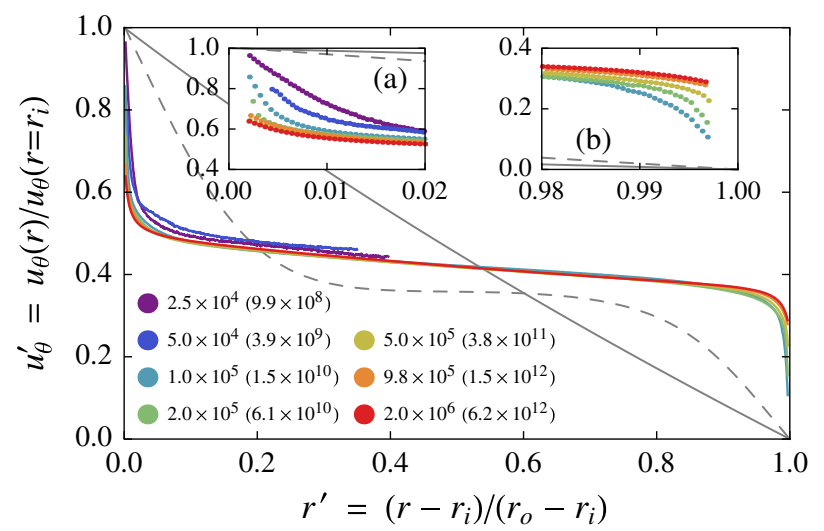

FIG. 2 (color online). Azimuthal velocity profiles for varying $\mathrm{Re}(\mathrm{Ta})$ across the gap of the TC apparatus. The legend indicates $\operatorname{Re}(\mathrm{Ta})$ of the experiments. Insets (a) and (b) show a zoom of the data of the inner and outer BL, respectively. Profiles in inset (a) are stacked top to bottom for increasing Ta. Individual data points are plotted in the insets showing the high resolution of the measurements. The gray solid line represents the exact laminar circular-Couette (nonvortical) solution of the NavierStokes equations. The dashed line is the profile for $\operatorname{Re}(\mathrm{Ta})=$ $260\left(10^{5}\right)$ obtained from DNS [21]. 
shallower slope due to turbulent mixing as the bulk flow in our parameter regime is fully turbulent $[8,30]$. As a comparison, we included the profile for one of the first vortical flows, $\operatorname{Re}=260$ [21]. For the laminar axially independent solution, the convective part of $J_{\omega}$ is zero; any convection in the bulk of the system therefore decreases the $\partial_{r} \omega$ term in the bulk, resulting in a shallower angular velocity gradient. From the insets we can clearly see that for increasing $\operatorname{Re}(\mathrm{Ta})$ the BLs become steeper, and indeed the angular velocity profile has then to become steeper as $u_{r}$ in $J_{\omega}$ is zero at the wall, and we are only left with the term $\partial_{r} \omega$. We now split our data in two parts: $r^{\prime} \in[0,1 / 2]$ (inner BL) and $r^{\prime} \in[1 / 2,1]$ (outer BL) and normalize velocities with the appropriate $u_{\tau}$ [Eqs. (3) and (6)] and distances with the respective $\delta_{\nu}$ [Eqs. (4) and (7)], see Fig. 3. We used global torque measurements $[8,37]$ to find $u_{\tau}$ and $\delta_{\nu}$. We spatially resolved the viscous sublayer $\left(y^{+}<5\right)$ for the lowest Re. It is known that in this viscous sublayer the velocity profile follows $u^{+}=y^{+}$. This concept also applies to TC flow, as supported by numerical simulations at low $\operatorname{Re}(\mathrm{Ta})$ (see, e.g., Ref. [21]). It should be noted that global torque measurements provide an average torque $\mathcal{T}$, while the local torque depends on height (following the large-scale Taylor vortex structure). So while Eq. (2) holds for the average $\tau_{w, i}$, it might have an axial dependence. However, it has been found [11] that the azimuthal velocity at midgap only has a weak axial dependence of the order of $\sim 1 \%$. To normalize the velocity profiles, we used the globally measured torque [as only this quantity is available for all $\operatorname{Re}(\mathrm{Ta})$ with sufficient precision] and thus the average $\tau_{w, i}$; this causes the imperfect matching of $u^{+}=y^{+}$.

In flat turbulent BL flows, at $y^{+}>50$ the effects of viscosity diminish. Furthermore, as suggested by Prandtl and von Kármán [45], in this limit the velocity profile converges to

$$
u^{+}=\frac{1}{\kappa} \ln y^{+}+B,
$$

with $\kappa$ the von Kármán constant and $B$ the logarithmic intercept. We will now apply this concept to TC flow.

Outside the viscous wall region $y^{+}>50$ (inside the outer layer), Fig. 3 shows the existence of a $\log$ layer in the ultimate TC regime ( $\mathrm{Ta} \gtrsim 10^{9}$ ), which is in sharp contrast to the laminar BLs found in the direct numerical simulations (DNS) [21] (indicated by thin lines) in the classical turbulent regime. Our profiles are fitted to Eq. (8) over the interval $y^{+} \in[50,600]$ (see the shaded area in Fig. 3). We have chosen $y^{+}=50$ as the lower edge of the interval as it is the start of the outer region, and the upper edge $y^{+}=600$ corresponds to about midgap $\left[\left(r_{i}+r_{o}\right) / 2\right]$ for our lowest $\mathrm{Re}(\mathrm{Ta})$ and the edge of the log regime for our highest Re (Ta) (see Fig. 3), but we stress that the values for $\kappa$ and $B$ only weakly depend on the exact extension of the fitting interval, as was already found for pipe flow [24]. The values for $\kappa$ are shown in Fig. 4. They are different for the inner and outer BL and depend on $\operatorname{Re}(\mathrm{Ta})$. For increasing $\operatorname{Re}(\mathrm{Ta})$, we see that the values for the inner BL approach a value of $\kappa \approx$ 0.40 , close to the known classical value of $\kappa=0.40$ [25,28], recently systematically examined by Marusic et al. [24].

For the smaller $\operatorname{Re}(\mathrm{Ta})$, the value for $\kappa$ is larger as the log layer is not yet fully developed, as clearly seen from Fig. 3. However, also geometric effects, namely, the curvature of the cylinder, may contribute to this deviation. To quantify this effect, we plot the ratio of the outer edge $600 \delta_{\nu}$ of the fitting regime to the radius of curvature of the cylinder (see Fig. 4) from which we conclude that for high $\operatorname{Re}>10^{6}$, the effect of the curvature becomes less than
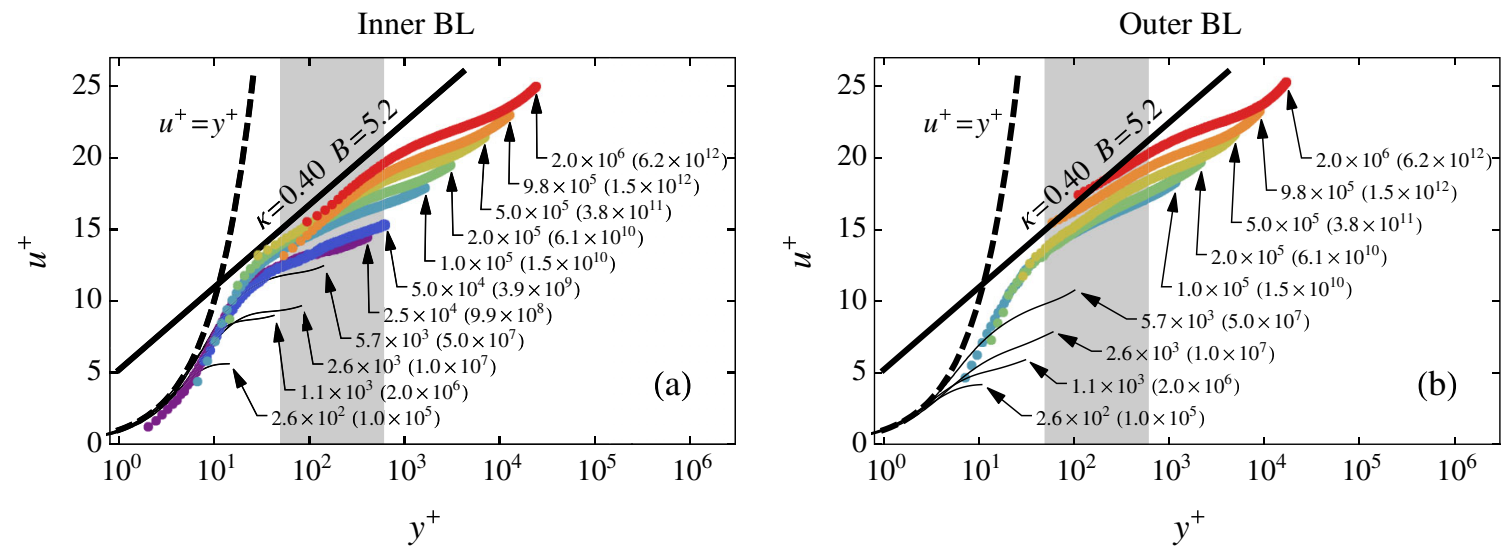

FIG. 3 (color online). (a) Azimuthal velocity profile near the inner cylinder $\left(r^{\prime} \in[0,1 / 2]\right)$ for varying $\operatorname{Re}(\mathrm{Ta}) . y^{+}$is the distance from the inner cylinder in units of the viscous length scale $\delta_{\nu} \cdot u^{+}$is defined as $\left[u\left(r_{i}\right)-u(r)\right] / u_{\tau}$, where $u(r)$ is the azimuthal component of the velocity, and $u\left(r_{i}\right)$ is the azimuthal velocity of the inner cylinder. (b) Azimuthal velocity profile near the outer cylinder $r^{\prime} \in[1 / 2,1] . y^{+}=\left(r_{o}-r\right) / \delta_{\nu}$ is the distance from the outer cylinder in wall units. For the case of the outer BL, the velocity is scaled as $u^{+}=u(r) / u_{\tau}$. Both figures include the logarithmic law of the wall $u^{+}=1 / \kappa \ln y^{+}+B$ by von Kármán, with the typical values of $\kappa=0.40$ and $B=5.2$, the viscous sublayer $u^{+}=y^{+}$, and in gray the fitting domain $y^{+} \in[50,600]$. Each data set is accompanied by an arrow with caption indicating $\operatorname{Re}(\mathrm{Ta})$. Thin solid lines are DNS results [21]. 


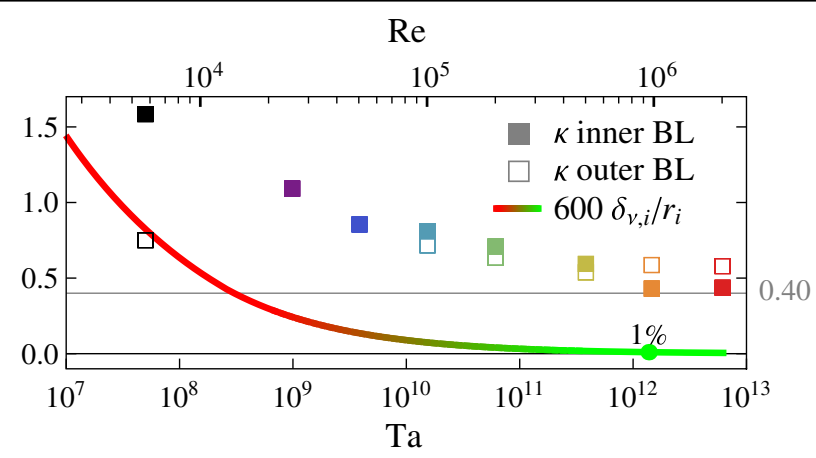

FIG. 4 (color online). The parameter $\kappa$ obtained by fitting $u^{+}=1 / \kappa \ln y^{+}+B$ for $y^{+} \in[50,600]$ near the inner and outer cylinder. The color scheme is identical to Figs. 2 and 3, the black symbols are DNS results [21]. The solid colored line shows the ratio of the upper edge $600 \delta_{\nu}$ of the fitting regime to the radius of curvature of the cylinder, quantifying the relative influence of the curvature. Note that $\delta_{\nu, i} / r_{i}=\delta_{\nu, o} / r_{o}$.

$1 \%$, i.e., negligible. Finally, we note that due to a possible slight height dependence of $\tau_{w, i}$ caused by the Taylor vortices even for this very turbulent flow, and the resulting imperfect matching of $u^{+}=y^{+}$, the values of $\kappa$ could still slightly vary with height.

In addition to PIV, high-resolution PTV measurements and analysis have also been performed. For these measurements, we zoomed into the area near the inner cylinder using a long-distance microscope to obtain a scaling factor of $\approx 10 \mu \mathrm{m} / \mathrm{px}$. The spatial resolution of PTV only depends on the number of images and can thus be better than the pixel grid spacing projected into physical space [31]. We extract the variance $\sigma^{2}\left(u_{\theta}\right)$ from the shape of the probability density function of the correlation function [33], see Fig. 5. We normalize $\sigma\left(u_{\theta}\right)^{2}$ with the friction velocity $u_{\tau}$ [see Fig. 5(a)] and with the driving velocity $u\left(r_{i}\right)$ [see Fig. 5(b)]. For both curves, the maximum of $\sigma\left(u_{\theta}\right)^{2}$ is around $y^{+}=12$, which is remarkably similar to the values obtained in pipe and channel flows (see, e.g., Refs. [27,28]). In addition, it can be observed that the collapse of the data is better when we normalize $\sigma\left(u_{\theta}\right)$ with the driving velocity rather than the shear velocity $u_{\tau}$. Opposed to the profiles shown in Fig. 3, in the outer layer $\left(y^{+}>50\right)$ the variance profiles universally collapse inside the buffer layer $\left(5<y^{+}<30\right)$ and seem not to depend on $\operatorname{Re}(\mathrm{Ta})$, vortical structure, and streamwise curvature. As shown in Fig. 5(a), we fit the data for $y^{+}>50$ (outer layer) with $\left[\sigma\left(u_{\theta}\right) / u_{\tau}\right]^{2}=B_{1}-A_{1} \ln y^{+}$-the log law for the velocity variance [25]. The corresponding fitting parameters are indicated in Fig. 5(a). Remarkably, the slope $A_{1}$ (varying from 1.26 to 1.45 ) is comparable with values found $\left(A_{1} \approx 1.25\right)$ in high Re BL flows [25].

To summarize, we performed direct measurements of the velocity BL profiles in highly turbulent TC flow up to $\mathrm{Ta}=6.2 \times 10^{12}$ and found the emergence of a log layer, as theoretically proposed in Refs. [3,4]. The fitted von Kármán constant $\kappa$ was found to approach the classical
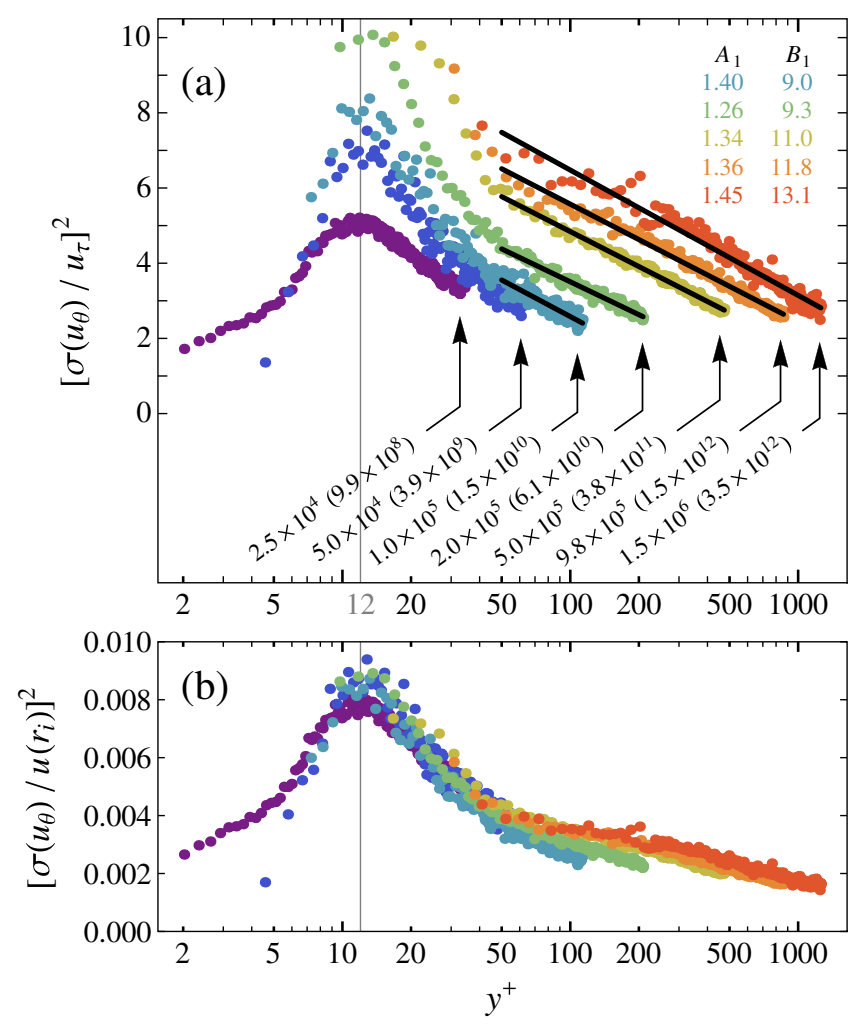

FIG. 5 (color online). (a) The variance of the local azimuthal velocity is presented as a function of radial distance from the inner cylinder. The velocity is made dimensionless using the friction velocity $u_{\tau}$, and the distance is in wall units $y^{+}=(r-$ $\left.r_{i}\right) / \delta_{\nu}$. (b) Same as in (a) but the velocity has been rescaled using the driving velocity $u\left(r_{i}\right)$. Corresponding colors in (a) and (b) correspond to the same data set and are consistent with previous figures. The $\operatorname{Re}(\mathrm{Ta})$ are indicated with arrows in (a). For $y^{+}>50$, the five highest Ta cases are fitted with $\left[\sigma\left(u_{\theta}\right) / u_{\tau}\right]^{2}=B_{1}-A_{1} \ln y^{+}$. The fitting parameters are listed inside (a) for increasing Ta and are colored accordingly.

value of 0.40 for large enough Ta. Furthermore, we found that the peak in $\sigma\left(u_{\theta}\right)$ universally collapsed around $y^{+}=12$, and that the height of the peak was found to collapse better when scaled with the driving velocity as compared to the friction velocity. Lastly, the variance profiles depicted a $\log$ dependence for $y^{+}>50$.

We would like to thank B. Benschop, M. Bos, and G. W. Bruggert for their technical support. We also acknowledge stimulating discussions with B. Eckhardt, S. Grossmann, M. Hultmark, I. Marusic, P. Monkewitz, A. J. Smits, and R. Verzicco. We thank R. Ostilla for making his DNS data available to us. This study was financially supported by the Simon Stevin Prize of the Technology Foundation STW of the Netherlands and the COST Action program MP0806: Particles in turbulence. The high-resolution PIV and PTV approaches were developed with financial support from the German Research Foundation (DFG) in the framework of the SFB-TRR 40 and the Individual Grants Programme KA $1808 / 8$. 
[1] C.D. Andereck, S.S. Liu, and H.L. Swinney, J. Fluid Mech. 164, 155 (1986).

[2] S. Grossmann and D. Lohse, J. Fluid Mech. 407, 27 (2000).

[3] R. H. Kraichnan, Phys. Fluids 5, 1374 (1962).

[4] S. Grossmann and D. Lohse, Phys. Fluids 23, 045108 (2011).

[5] S. Grossmann and D. Lohse, Phys. Fluids 24, 125103 (2012).

[6] X. He, D. Funfschilling, H. Nobach, E. Bodenschatz, and G. Ahlers, Phys. Rev. Lett. 108, 024502 (2012).

[7] G. I. Taylor, Phil. Trans. R. Soc. A 223, 289 (1923).

[8] G. S. Lewis and H. L. Swinney, Phys. Rev. E 59, 5457 (1999).

[9] S. Huisman, C. Sun, and D. Lohse (unpublished).

[10] D. P. Lathrop, J. Fineberg, and H. L. Swinney, Phys. Rev. Lett. 68, 1515 (1992).

[11] D. P. M. van Gils, S. G. Huisman, S. Grossmann, C. Sun, and D. Lohse, J. Fluid Mech. 706, 118 (2012).

[12] C. Sun, Y. H. Cheung, and K. Q. Xia, J. Fluid Mech. 605, 79 (2008).

[13] G. Ahlers, S. Grossmann, and D. Lohse, Rev. Mod. Phys. 81, 503 (2009).

[14] D. Lohse and K. Q. Xia, Annu. Rev. Fluid Mech. 42, 335 (2010).

[15] Q. Zhou and K.Q. Xia, Phys. Rev. Lett. 104, 104301 (2010).

[16] F. Wendt, Arch. Appl. Mech. 4, 577 (1933).

[17] G. P. Smith and A. A. Townsend, J. Fluid Mech. 123, 187 (1982).

[18] S. T. Wereley and R. M. Lueptow, J. Fluid Mech. 364, 59 (1998).

[19] S. Dong, J. Fluid Mech. 587, 1 (2007).

[20] R. van Hout and J. Katz, Phys. Fluids 23, 105102 (2011).

[21] R. Ostilla, R. J. A. M. Stevens, S. Grossmann, R. Verzicco, and D. Lohse, J. Fluid Mech. 719, 14 (2013).

[22] H. J. Brauckmann and B. Eckhardt, J. Fluid Mech. 718, 398 (2013).

[23] G. Ahlers, E. Bodenschatz, D. Funfschilling, S. Grossmann, X. He, D. Lohse, R. J. A. M. Stevens, and R. Verzicco, Phys. Rev. Lett. 109, 114501 (2012).

[24] I. Marusic, J. P. Monty, M. Hultmark, and A. J. Smits, J. Fluid Mech. 716, R3 (2013).

[25] C. Meneveau and I. Marusic, J. Fluid Mech. 719, R1 (2013).
[26] T. Wei and W.W. Willmarth, J. Fluid Mech. 204, 57 (1989).

[27] I. Marusic, B. J. McKeon, P. A. Monkewitz, H. M. Nagib, A. J. Smits, and K. R. Sreenivasan, Phys. Fluids 22, 065103 (2010).

[28] M. Hultmark, M. Vallikivi, S. C. C. Bailey, and A. J. Smits, Phys. Rev. Lett. 108, 094501 (2012).

[29] D. P. Lathrop, J. Fineberg, and H. L. Swinney, Phys. Rev. A 46, 6390 (1992).

[30] S. G. Huisman, D. P. M. van Gils, S. Grossmann, C. Sun, and D. Lohse, Phys. Rev. Lett. 108, 024501 (2012).

[31] C. J. Kähler, S. Scharnowski, and C. Cierpka, Exp. Fluids 52, 1629 (2012).

[32] C. J. Kähler, S. Scharnowski, and C. Cierpka, Exp. Fluids 52, 1641 (2012).

[33] S. Scharnowski, R. Hain, and C. J. Kähler, Exp. Fluids 52, 985 (2012).

[34] B. Eckhardt, S. Grossmann, and D. Lohse, J. Fluid Mech. 581, 221 (2007).

[35] H. Ji, M. Burin, E. Schartman, and J. Goodman, Nature (London) 444, 343 (2006).

[36] M.S. Paoletti and D. P. Lathrop, Phys. Rev. Lett. 106, 024501 (2011).

[37] D. P. M. van Gils, S. G. Huisman, G. W. Bruggert, C. Sun, and D. Lohse, Phys. Rev. Lett. 106, 024502 (2011).

[38] S. Merbold, H. J. Brauckmann, and C. Egbers, Phys. Rev. E 87, 023014 (2013).

[39] D. P. M. van Gils, G. W. Bruggert, D. P. Lathrop, C. Sun, and D. Lohse, Rev. Sci. Instrum. 82, 025105 (2011).

[40] Dantec, FPP-RHB-10, fluorescent polymer tracer particles, 1-20 $\mu \mathrm{m}$, water suspension.

[41] InnoLas, Flash lamp-pumped Nd:YAG laser, SpitLight 400.

[42] pco, pco.edge camera, $2560 \mathrm{px} \times 2160 \mathrm{px}$ resolution with $6.5 \mu \mathrm{m}$ pixel size, in front a $50 \mathrm{~mm}$ objective lens is mounted with a working distance of approximately $600 \mathrm{~mm}$, giving a scaling factor of $\approx 54 \mu \mathrm{m} / \mathrm{px}$.

[43] C. J. Kähler, U. Scholz, and J. Ortmanns, Exp. Fluids 41, 327 (2006).

[44] Infinity, K2 long-distance microscope, focal length of $300 \mathrm{~mm}$.

[45] S. B. Pope, Turbulent Flows (Cambridge University Press, Cambridge, England, 2000). 